\title{
Benefits of social vs. non-social feedback on learning and generosity. Results from theTipping Game
}

\author{
Matteo Colombo $^{1 * t}{ }^{\dagger}$, Aistis Stankevicius ${ }^{2 \dagger}$ and Peggy Seriès ${ }^{2}$ \\ 1 Tilburg Center for Logic, General Ethics, and Philosophy of Science, Tilburg University, Tilburg, Netherlands \\ 2 Institute for Adaptive and Neural Computation, University of Edinburgh, Edinburgh, UK
}

\author{
Edited by: \\ Giulia Andrighetto, Institute of \\ Cognitive Science and Technologies - \\ National Research Council, Italy \\ Reviewed by: \\ David R. Simmons, University of \\ Glasgow, UK \\ Aron Szekely, University of Oxford, UK \\ *Correspondence: \\ Matteo Colombo, Tilburg Center for \\ Logic, General Ethics, and Philosophy \\ of Science, Tilburg University, \\ P. O. Box 90153, 5000 LE \\ Tilburg, Netherlands \\ e-mail:m.colombo@uvt.nl \\ ${ }^{\dagger}$ Matteo Colombo and Aistis \\ Stankevicius have contributed equally \\ to this work.
}

Although much work has recently been directed at understanding social decision-making, relatively little is known about how different types of feedback impact adaptive changes in social behavior. To address this issue quantitatively, we designed a novel associative learning task called the "Tipping Game," in which participants had to learn a social norm of tipping in restaurants. Participants were found to make more generous decisions from feedback in the form of facial expressions, in comparison to feedback in the form of symbols such as ticks and crosses. Furthermore, more participants displayed learning in the condition where they received social feedback than participants in the nonsocial condition. Modeling results showed that the pattern of performance displayed by participants receiving social feedback could be explained by a lower sensitivity to economic costs.

\section{Keywords: social/non-social feedback, facial expressions, social norms, tipping behavior, associative learning}

\section{INTRODUCTION}

Several behavioral, neurobiological and theoretical studies have shown that social norm compliance, and more generally adaptive changes in social behavior, often require the effective use and weighing of different types of information, including expected economic costs and benefits, the potential impact of our behavior on the welfare of others and our own reputation, as well as feedback information (Bicchieri, 2006; Adolphs, 2009; Frith and Frith, 2012). Relatively little attention has been paid to how different types of feedback (or reward) may impact the way social norms are learned. The present study addresses this issue with behavioral and modeling results from a novel associative learning task called the "Tipping Game." We take the example of tipping and ask: how do social feedback in the form of facial expressions, as opposed to non-social feedback in the form of such conventional signs as ticks and crosses, affect the way participants learn a social norm of tipping?

Recent findings indicate that people's decision-making is often biased by social stimuli. For example, images of a pair of eyes can significantly increase pro-social behavior in laboratory conditions as well as in real-world contexts (Haley and Fessler, 2005; Bateson et al., 2006; Rigdon et al., 2009; Ernest-Jones et al., 2011). Furthermore, decision-making can be systematically biased by facial emotional expressions used as predictors of monetary reward (Averbeck and Duchaine, 2009; Evans etal., 2011; Shore and Heerey, 2011). Facial expressions of happiness elicit approaching behavior, whereas angry faces elicit avoidance (Seidel et al., 2010; for a review see Blair, 2003). Because they can function as signals to others, eliciting specific behavioral responses, emotional facial expressions play a major role in socialization practices that help individuals to adapt to the norms and values of their culture (Keltner and Haidt, 1999; Frith, 2009).

Despite this body of findings, the literature does not provide an unambiguous answer to the question of how learning performance is affected by social stimuli in comparison to different types of non-social stimuli used as feedback about previous decisions in a learning task (Ruff and Fehr, 2014). Consistent with the view that social reinforcement is a powerful facilitator of human learning (Zajonc, 1965; Bandura, 1977), one recent study using a feedback-guided item-category association task found that learning performance in control groups was improved when social (smiling or angry faces) instead of nonsocial (green or red lights) reinforcement was used (Hurlemann et al., 2010).

However, the paradigm used in this study did not distinguish between two conditions in which social-facilitative effects on learning performance have been observed: first, a condition characterized by the mere presence of others (Allport, 1920); and second, a condition where others provide reinforcing feedback (Zajonc, 1965). In the task used by Hurlemann et al. (2010), faces were present onscreen throughout each trial, changing from a neutral to a happy expression for correct responses or angry for incorrect responses. So, this study could not identify the specific effect of social feedback on learning.

Consistent with the assumption oft made in economics and psychology that optimal decisions and learning are based on an assessment of the evidence that is unbiased by the social or nonsocial nature of the evidence itself (Becker, 1976; Oaksford and Chater, 2007), Lin et al. (2012a) found that, instead of boosting learning performance, social reward (smiling or angry faces) made 
learning slower, and generally less effective, in comparison to nonsocial reward such as money.

It should be noted that Hurlemann et al. (2010) and Lin et al. (2012a) were examining fundamentally different questions, which may explain the difference in their results. In Hurlemann et al.'s (2010) study, participants used feedback to learn the category membership of an abstract string of numbers, whereas in Lin et al.'s (2012a) study participants played an instrumental learning task where they had to learn to select the slot machine associated with the highest probability of a positive valenced outcome. So, depending on the task, social stimuli may have different, sometimes opposite, effects on learning performance.

In particular, it remains controversial whether participants in an associative learning task receiving feedback in the form of facial expressions learn a social norm more effectively than participants who are provided with non-social, cognitive feedback.

Our study contributes to previous literature by examining more closely the relative impact of social (happy and angry faces) and non-social feedback (tick and cross marks) on learning, and by testing the hypothesis that social feedback leads to more generous behavior, in the context of the Tipping Game. This task tapped into a basic mechanism underlying the ontogeny of social cognition (Reeb-Sutherland et al., 2012), while allowing us to examine the effects of social, as opposed to non-social, feedback on learning and decision-making.

The Tipping Game shares several features with other reinforcement learning tasks, and so the associated modeling framework can be used to quantitatively characterize the behavioral results of both healthy young people_-as in our study - as well as clinical and neurological patients (Lin et al., 2012b). In the present study, modeling results helped to disentangle how information carried by specific types of feedback stimuli may interact with economic interest when people are learning a social norm. The originality of the Tipping Game is the social context and social feedback that it involves. These contribute to the higher ecological validity and naturalness of our task, which distinguish it from the ones previously used in studies using facial expressions as predictors of monetary reward (e.g., Averbeck and Duchaine, 2009; Hurlemann et al., 2010; on the importance of the ecological validity in these types of tasks, see Lin et al., 2012b, p. 7).

\section{EXPERIMENT 1 \\ METHODS \\ Participants}

Forty participants (23 males) made decisions in the Tipping Game. The participants were students in the University of Edinburgh, signed informed consent approved by the University of Edinburgh Ethics Committee, and were compensated with $\mathfrak{E} 6 / \mathrm{h}$ for taking part in the experiment. While some of the participants were international students, they all lived in the UK for 1 year or more.

\section{Design and procedure}

The Tipping Game was implemented in Matlab, using the Psychophysics Toolbox 3 extensions (Brainard, 1997; Pelli, 1997; Kleiner et al., 2007), and was run on computers in sound-deadened booths.
Initially, participants filled out five questionnaires: the "Empathy Quotient" (EQ) questionnaire (Baron-Cohen and Wheelwright, 2004), one version of the "Reading the Mind in the Eyes" test (Baron-Cohen etal., 1997), the "Self Report Altruism" questionnaire (Rushton et al., 1981), the "Sensitivity to Punishment and Sensitivity to Reward Questionnaire" (SPSRQ; Torrubia et al., 2001), and the "Behavioral Inhibition/Approach" (BIS/BAS) questionnaire (Carver and White, 1994). These questionnaires measured respectively the level of empathy, mentalizing, altruism, and punishment and reward sensitivity of the participants.

After the questionnaires were completed, instructions were given about the Tipping Game and its goals. Participants were informed that they would take part in a computer-based task where they had to imagine to be diners at restaurants in some unfamiliar country. In each trial of the task, they would be presented firstly with information regarding the quality of the service received at a restaurant (i.e., either good or bad service). Then, the bill to be paid would be disclosed. Finally, participants should decide which amount to tip in that restaurant taking into account the quality of the service. After each decision, either positive or negative feedback would be revealed, indicating that the tip was either higher or lower than expected. Whether positive or negative feedback was revealed depended stochastically on the underlying social norm and on the pair service quality - amount tipped (see Table 1). Feedback information received after a decision could be used by participants to learn how much they were expected to tip.

The task consisted of three blocks, each one of which comprised forty trials. A trial corresponded to one tipping interaction of the sort just described. At the beginning of each block, participants were endowed with 1,100 monetary units (mu), with which they had to pay for restaurant bills and any tip they decided to give. It was made explicit to the participants that the mu they were using were fictional, and that no actual person would benefit from their tips. All participants knew that their goal was to learn a social norm of tipping so as to display adaptive behavior in the social situation they were facing, while saving as much mu as possible.

To motivate participants to pursue this goal, they were told at the beginning of the task that the best overall performance would be rewarded with $\mathfrak{E} 20$, and that their overall performance would be measured as a function of both how well the social norm was learned and how much mu was saved across the whole task.

Table 1 | Feedback structure in Experiment 1.

\begin{tabular}{llll}
\hline $\begin{array}{l}\text { Probability of positive } \\
\text { feedback }\end{array}$ & $\begin{array}{l}\text { Block 1 } \\
\text { (norm 23\%) }\end{array}$ & $\begin{array}{l}\text { Block 2 } \\
\text { (norm 50\%) }\end{array}$ & $\begin{array}{l}\text { Block 3 } \\
\text { (norm 23\%) }\end{array}$ \\
\hline Good service & $20 / 80$ & $20 / 80$ & $35 / 65$ \\
Bad service & $30 / 70$ & $30 / 70$ & $40 / 60$
\end{tabular}

Probability of obtaining a positive feedback as a function of the action that was made (tip < norm/tip $\geq$ norm), and in the situation where the service was good (first line) or bad (second line). 
Participants were also informed that some manipulation could take place across blocks so that they should treat each block as a novel kind of situation (i.e., a different unfamiliar country).

Two types of manipulations took place. First, the underlying social norm of tipping changed between blocks. Second, the reliability of the reward feedback changed across blocks, with feedback being more random in the third block than in the previous blocks (Table 1).

In one condition (Social Condition), 20 participants (seven females) received feedback in the form of a happy or an angry face. Pictures of facial expressions were in greyscale and selected from the Japanese Female Facial Expression (JAFFE) database (Lyons et al., 1998). In a second condition (non-social condition), 20 participants (10 females) received non-social feedback in the form of a tick (also known as a check mark) or an X mark in greyscale.

Participants filled out a debriefing questionnaire after they completed the task.

\section{Results and discussion}

All data analyses in this and the following experiments were performed off-line using commercial software packages SPSS 22 (IBM Corp., 2013) and MATLAB 8.1 (MathWorks Inc., 2013).

The results of the personality questionnaire showed that all participants displayed normal capacity for recognition of facial expressions. On average, they also presented normal levels of altruism, empathy and attitudes toward reward and punishment (Table 2).

Results from the debriefing questionnaires indicated that all participants recognized the symbols used in the non-social condition as the notation respectively for positive and negative feedback. One participant reported not having understood the task so his data was excluded from further analysis.

Changes in tipping behavior for the two conditions during the task were tested using the multiple analysis of covariance (MANCOVA) procedure, with tipping percentages in each block (three blocks) as dependent variables, group (two groups) as a between subjects factor, and trial number (1-40) as a covariate. The analysis was run on data concerning the decisions of 39 participants (20 and 19 participants for social and non-social conditions respectively) in the two groups.

Social feedback was found to have a different impact on tipping and learning than non-social reward. Specifically, social feedback was found to have a significant effect on the percentages tipped in blocks 2 and 3 (Table 3 ). Furthermore, considering the whole task, the difference between the social norm and the amount tipped by participants in the social condition was significantly lower than the difference between the social norm and the amount tipped by participants in the non-social condition, i.e., the social group tipped more by 1, 4.9 and $8.2 \%$ in blocks 1,2 and 3 respectively. The first finding confirms the hypothesis that social feedback in the form of facial expressions led participants to display higher degree of generosity, viz. tipping contribution. The second finding confirms that learning is facilitated by social feedback.

A large inter-individual variability of performance was observed, with some participants learning to adapt their decisions to conform to the norm much better than other participants, who seemed to behave independently of the feedback throughout the experiment. Nonetheless, more participants displayed learning in the social condition than participants in the non-social condition. To quantify this, and identify the number of learners in the task, we assumed that if the mean amount tipped by a participant was significantly different from one block to the next and moved toward the underlying social norm, then that participant displayed learning. According to this criterion, we found that $12 / 20$ displayed learning in the social group, vs. 7/19 in the non-social group.

With respect to the scores of the personality questionnaires we administered, no significant difference between groups was found (see Table 2). This finding mitigates the concern that the behavioral effects observed could be explained solely by some stable personality trait-for instance, by some autistic trait.

Two further points should be noted. First, although the difference between the social norm and the amount tipped by participants in the social condition was significantly lower than that for participants in the non-social condition, participants in both groups always tipped less than the norm in the first two blocks. One hypothesis that could explain this finding is that participants had a strong prior bias toward a specific action different from the social norms in our task. Based on questionnaire results, it is plausible that participants, who all lived in the UK for 1 year or more, came to the Tipping Game with a prior of $13 \%$, which would be consistent with the behavior they displayed throughout the experiment. Second, standard deviations from average percentages tipped in both groups were high, especially in the third block (see Figure 1). Considering the whole experiment, the standard deviation from the average percentage tipped by the social

Table 2 | Personality questionnaires average scores for social (Soc.) and non-social (Non-Soc.) groups.

\begin{tabular}{llllllllll}
\hline & Faces & Altruism & Drive & FS & RR & BI & EQ & SR & SP \\
\hline Soc. & $17.9(1.98)$ & $55.3(10.32)$ & $10.3(1.62)$ & $11.9(3.00)$ & $15.7(2.38)$ & $21.3(3.49)$ & $39.0(14.97)$ & $10.9(3.91)$ & $11.4(5.36)$ \\
Non-Soc. & $18.2(1.09)$ & $56.1(9.01)$ & $10.9(1.92)$ & $11.6(2.08)$ & $17.5(2.14)$ & $21.4(2.85)$ & $45.7(7.92)$ & $9.9(3.71)$ & $10.2(4.35)$ \\
p-value & 0.548 & 0.796 & 0.263 & 0.796 & 0.019 & 0.866 & 0.092 & 0.417 & 0.454
\end{tabular}

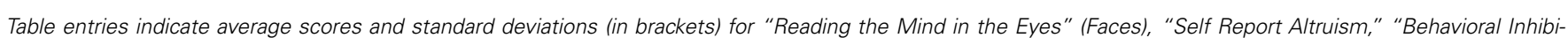

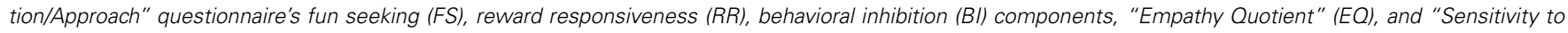

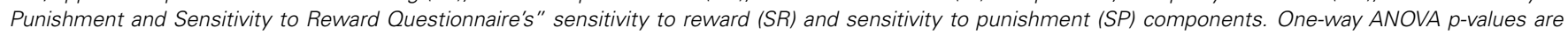

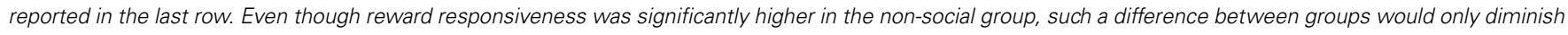
the effect size. 
Table 3 | Multiple analysis of covariance (MANCOVA) results for Experiment 1.

\begin{tabular}{|c|c|c|c|c|c|c|c|}
\hline Source & Dependent variable & Grand mean (\%) & Standard deviation (\%) & DoF & $\boldsymbol{F}$ & Significance & $\eta_{\mathrm{p}}^{2}$ \\
\hline \multirow[t]{2}{*}{ Group (IV) } & Block 1 & 14.8 & 12.3 & 1 & 2.526 & 0.112 & 0.002 \\
\hline & Block 3 & 23.3 & 68.1 & 1 & 5.653 & 0.018 & 0.004 \\
\hline \multirow[t]{2}{*}{ Error } & Block 1 & & & 1557 & & & \\
\hline & Block 2 & & & 1557 & & & \\
\hline
\end{tabular}

Testing was performed on the effects of the independent variable (IV) Group (i.e., the type of feedback received by participants) on the percentage of the bill tipped by participants. The mean tipping percentage (i.e., percentage of the bill tipped) and standard deviation for each block is presented with corresponding degrees of freedom (DoF), F statistics, significance ( $p$-value), and effect size $\left(\eta_{p}^{2}\right)$.
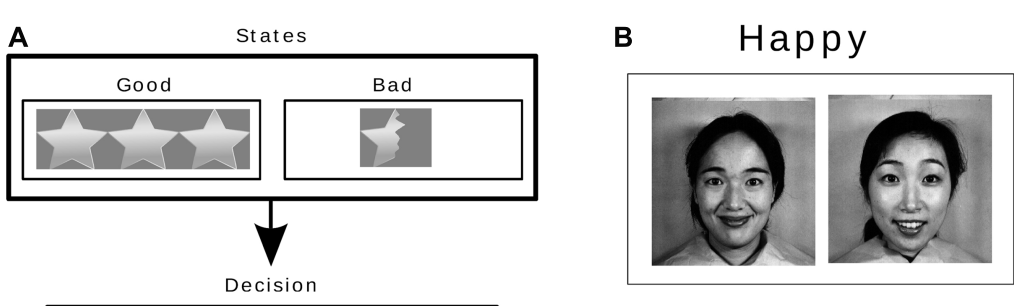

C

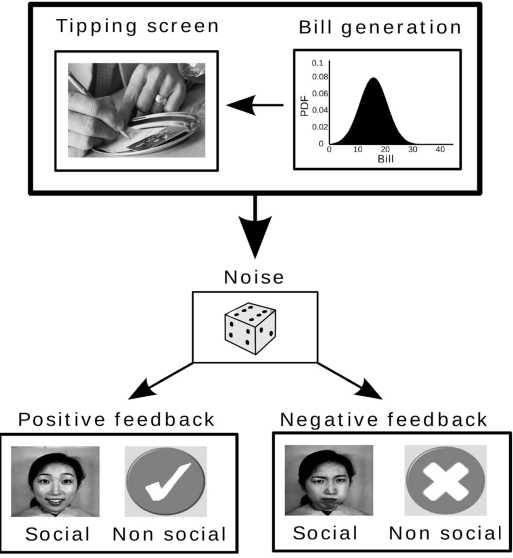

FIGURE 1 | (A) Structure of a single trial. First, state of service is presented (either good or bad). Then, the participant is presented with a bill drawn from a normal distribution with $\mu=18, \sigma=5$ (truncated to be $>3$ and $<45$ ). A decision is made, and the participant is presented with either positive or negative feedback (with a certain probability; see

Tables $\mathbf{1}$ and $\mathbf{4}$ for details) represented as a face or a tick/cross for social and non-social conditions respectively. The task was implemented using the Matlab Psychophysics Toolbox extensions. (B) Faces used as social feedback cues (Lyons etal., 1998). (C) Group averaged tipping performances in Experiment 1 (top) and Experiment 2 (bottom). Shaded areas are \pm standard deviation; the dashed line is the social norm of tipping. group was 5.64 vs. $3.39 \mathrm{mu}$ for the non-social group. So, in general, we observed high variability in the behavioral data; in particular, participants in the social group took actions that were spread out over a larger range of values in comparison to the actions taken by participants in the non-social group.

\section{EXPERIMENT 2}

Results from Experiment 1 raised two questions. First, does social feedback impact both learning performance and willingness to be generous to the same degree, or rather one of these two factors more than the other? Experiment 1 could not answer this question because the norms of tipping to be learned were above standard real-life levels (i.e., around 15\%). So, learning performance aligned with generosity. If social feedback boosts generosity more than learning, then it will lead to a resistance toward learning a new norm when this norm is lower than the standard; that is, learning performance will be biased toward over-tipping.

Second, does social feedback impact learning performance only when the difficulty of the task is higher? Results from Experiment 1 could be explained simply as a function of the difficulty of the task, namely: the more unreliable was the feedback, the more significant was the difference we found between social and non-social group. If social feedback is effective only when the task is difficult, then 
no significant difference will be found between groups when the feedback provided is more reliable. Experiment 2 was designed to test these two predictions.

\section{METHODS}

\section{Participants}

A novel sample of forty participants (12 males) made decisions in the Tipping Game. All participants were students in the University of Edinburgh, signed informed consent approved by the University of Edinburgh Ethics Committee and were compensated with $\mathfrak{E} 6 / \mathrm{h}$ for taking part in the experiment. While some of the participants were international students, they all lived in the UK for 1 year or more.

\section{Design and procedure}

This second experiment was different from Experiment 1 in three respects. To test our first prediction, the social norm of tipping in the second block was lower than the standard: it was 7\%. To test our second prediction, we added a fourth block, where the reliability of the feedback was higher (see Table 4). Including a fourth block, the task in this second experiment was longer than in Experiment 1. So, in order to prevent participants from getting excessively bored or fatigued, we decided not to administer personality questionnaires.

\section{Results and discussion}

Results from the debriefing questionnaires indicated that all participants recognized the symbols used in the non-social condition as the notation respectively for positive and negative feedback.
Changes in tipping behavior for the two conditions during the task were tested using the MANCOVA procedure, with tipping percentages in each block (four blocks) as dependent variables, group (two groups) as a between subjects factor, and trial number (1-40) as a covariate. The analysis was run on data concerning the decisions of 40 in the two groups.

Social feedback was found to have a different impact on learning and social decision-making than non-social feedback, thus confirming the results of Experiment 1. Specifically, social feedback cues were found to have a significant effect on percentages tipped in all blocks (see Table 5). Furthermore, the difference between the social norm and the amount tipped by participants in the social condition was significantly lower than the difference between the social norm and the amount tipped by participants in the non-social condition, i.e., social group tipped more by $7.2,1.8,2.9$ and $10.6 \%$ in blocks 1 to 4 respectively.

So, as the percentages tipped by the social group were found to be higher than those of the non-social group across all blocks, the hypothesis receives some confirmation that social feedback in the form of facial expressions led participants to display higher degrees of generosity. As in experiment 1, the second difference we found indicates that social cues may facilitate learning of a social norm. Although in block 2 the social group did not learn the norm better than the non-social group, which suggests that the learning effect observed in all other conditions might be driven by generosity, more participants displayed learning in the social condition than in the non-social condition. According to

Table 4 | Feedback structure in Experiment 2.

\begin{tabular}{lllll}
\hline $\begin{array}{l}\text { Probability of positive feedback } \\
\text { (tip }<\text { norm/tip } \geq \text { norm) }\end{array}$ & Block $\mathbf{1}$ (norm 23\%) & Block 2 (norm 7\%) & Block 3 (norm 23\%) & Block $\mathbf{4}$ (norm 50\%) \\
\hline Good state & & & $35 / 65$ & $5 / 95$ \\
Bad state & $20 / 80$ & $20 / 80$ & $40 / 60$ & $10 / 90$ \\
\hline
\end{tabular}

Probability of obtaining a positive feedback as a function of the action that was made (tip $<$ norm/tip $\geq$ norm), and in the situation where the service was either good (first line) or bad (second line).

Table 5 | Multiple analysis of covariance (MANCOVA) results for Experiment 2.

\begin{tabular}{|c|c|c|c|c|c|c|c|}
\hline Source & Dependent variable & Grand mean & Standard deviation & DoF & $F$ & Significance & $\eta_{p}^{2}$ \\
\hline \multirow[t]{3}{*}{ Group (IV) } & Block 1 & $19.8 \%$ & $16.8 \%$ & 1 & 77.006 & 0 & 0.046 \\
\hline & Block 3 & $21.9 \%$ & $17.6 \%$ & 1 & 10.810 & 0.001 & 0.007 \\
\hline & Block 4 & $31.2 \%$ & $20.7 \%$ & 1 & 117.125 & 0.000 & 0.068 \\
\hline \multirow{2}{*}{ Error } & Block 3 & & & 1597 & & & \\
\hline & Block 4 & & & 1597 & & & \\
\hline
\end{tabular}

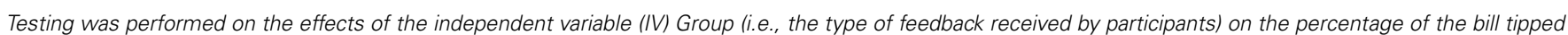

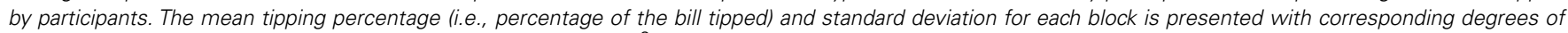

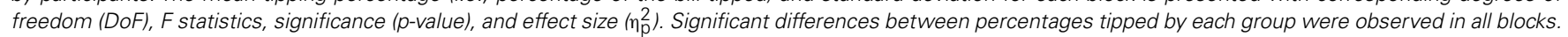


the learning criterion described above, we found that $17 / 20$ displayed learning in the social group, vs. 10/20 in the non-social group.

\section{A MODEL OF THE TIPPING GAME}

To further describe quantitatively the nature of the effects that we observed, we used a Rescorla-Wagner reinforcement learning algorithm (Rescorla and Wagner, 1972) to model the behavior of participants in our two experiments. The model algorithm makes decisions in our task with the goal of maximizing its total reward. It does this by learning action values $Q$ for state-action pairs, and selecting actions in function of their estimated $Q$-values at each trial.

The possible states were two, corresponding to "good" or "bad" service quality. The action space comprised 101 actions, corresponding to tip percentages from $0 \%$ increasing in steps of 1 to $100 \%$. For each of the two states, the action taken by the model was iteratively assigned a value $Q$, which was a function of the reward obtained for taking that action (a combination of feedback received and economic cost incurred, see below) and the $Q$-value of that state-action pair stored in memory. This is expressed by the Q-update equation:

$$
\begin{aligned}
Q(\text { state }, \text { action })_{\text {new }}= & Q(\text { state }, \text { action })_{\text {old }}+ \\
& \alpha\left(\text { reward }-Q(\text { state, action })_{\text {old }}\right)
\end{aligned}
$$

where $\alpha$ is the learning rate $(0 \leq \alpha \leq 1)$. The smaller $\alpha$, the less the existing knowledge is modified. Conversely, as $\alpha$ tends to 1 , what has already been learned can be quickly overwritten. The reward signal in our model consisted of the hyperbolic tangent (scaling to interval $\left[\begin{array}{ll}-1 & 1\end{array}\right]$ ) of the weighted difference of two components: an economic component and a reward outcome component. Formally:

$$
\text { Reward }=\tanh \left(w_{\text {out }} r_{\text {out }}-w_{\text {econ }} r_{\text {econ }}\right)
$$

where $r_{\text {econ }}$ is an economic factor that is equal to the Tip/Bill ratio. As we assumed that the model could tip at most an amount equal to the bill, the range of this economic factor is $0 \leq r_{\text {econ }} \leq 1$. The economic weight $w_{\text {econ }}$ is a parameter that determines the extent to which spending money in tips was valued in the Tipping Game. We assumed that, at best, spending money in tips would not have any negative impact on an agent's reward signal, and we set the range of this parameter to $0 \leq w_{\text {econ }} \leq w_{\max }$, where $w_{\max }$ is 10 . So, lower values of $w_{\text {econ }}$ indicate that the agent does not mind spending money in tips. Conversely, higher values of $w_{\text {econ }}$ indicate that the agent does mind spending money in tips. If the agent does not mind spending money in tips at all (viz., $w_{\text {econ }}=0$ ), then the economic cost of a tip, whatever its amount, will not weigh against the agent's overall reward. So, if the agent displays $w_{\text {econ }}=0$, then it will systematically over-tip in the Tipping Game. Roughly, lower values of $w_{\text {econ }}$ correspond to a more nonchalant attitude toward tipping — whatever the amount. The reward outcome factor $r_{\text {out }}$ is associated to the two possible outcomes in the task: either positive feedback or negative feedback. The reward magnitude of positive feedback is assumed to be 1 , while the magnitude of negative feedback is assumed to be
0 . The outcome weight $w_{\text {out }}\left(0 \leq w_{\text {out }} \leq w_{\max }\right)$ is a parameter that determines to what extent positive feedback was valued. Higher values of $w_{\text {out }}$ correspond to a more positive feedbackseeking attitude. When $w_{\text {econ }}>w_{\text {out }}$, it should be expected that the agent will severely under-tip in the Tipping Game. When $w_{\text {econ }}<w_{\text {out }}$, the learning behavior of the agent in the Tipping Game will depend heavily on the action selection mechanism, in particular on how the exploration of the action space is performed.

Finally, the model made choices according to the "softmax" rule:

$$
p(\text { action } \mid \text { state })=\frac{\exp (\tau Q(\text { state }, \text { action }))}{\sum_{i=1}^{N} \exp \left(\tau Q\left(\text { state }_{\text {action }}\right)\right)}
$$

which determines the probability of selecting a certain action (e.g., a tip of $11 \%$ of the bill) for a given state (e.g., "good" service). The parameter $\tau$ featuring in (3) is positive and is called "inverse temperature." A low $\tau$ causes the actions to be all nearly equiprobable; whereas, as $\tau$ gets larger, the action with the highest expected reward has a much higher probability of being selected than the other actions.

\section{MODELING RESULTS}

To estimate parameters values, we fitted the model to each participant using maximum likelihood, resulting in a set of parameters (i.e., $\alpha, \tau, w_{\text {out }}$ and $w_{\text {econ }}$ ) that maximized the probability that the model-agent would make the same choices as the participant (on average over the course of the experiment).

The parameter values obtained confirmed that participants in the social group displayed different learning and decision-making profiles from participants in the non-social group. For the two experiments, a large difference between the social and non-social group was found in the economic weight $w_{\text {econ }}$ : in comparison to participants in the non-social condition, participants in the social condition were much less sensitive to economic costs, thereby displaying a more generous tipping behavior (Table 6). This difference is partly explained by the number of learners in each group. Smaller, but non-zero, values of $w_{\text {econ }}$ were characteristic of participants who displayed a better learning performance, and, according to the learning criterion we employed (see Results and Discussion), the number of learners in the social group was larger than the number of learners in the non-social group. So, it should be expected that the larger the difference in the number of learners

Table 6 | Average model parameters for the two experiments.

\begin{tabular}{llllll}
\hline & Group & $\boldsymbol{\alpha}$ & $\boldsymbol{T}$ & $\boldsymbol{w}_{\text {out }}$ & $\boldsymbol{w}_{\text {econ }}$ \\
\hline Experiment 1 & Social & 0.24 & 93.2 & 4.43 & 0.004 \\
& Non-social & 0.2 & 78.8 & 3.82 & 0.038 \\
Experiment 2 & Social & 0.4 & 20.7 & 5.68 & 0.002 \\
& Non-social & 0.29 & 59.9 & 4.51 & 0.081
\end{tabular}

Significant differences $(p=0.019$ and $p=0.049$ in Experiments 1 and 2 respectively; independent two-tailed $t$-tests) were found in economic weight values between the groups ( $w_{\text {econ }}$ ). 
between the two groups, the larger is the difference between the economic weights $w_{\text {econ }}$ that characterize the two group's tipping behavior.

\section{GENERAL DISCUSSION}

Our study asked how the type of feedback obtained by people after they make decisions in social situations affect the way they learn a social norm. We addressed this question by determining whether the influence of facial expressions on participants' decisions in a novel associative learning task called the "Tipping Game" was significantly different from the influence of non-social feedback in the form of conventional marks. We found that participants receiving feedback in the form of happy or angry facial expressions behaved in a significantly different way than participants receiving feedback in the form of tick or cross marks. This effect was observed across most blocks in our task, and, specifically, had impact on how much participants were willing to give as a tip and on how well they learned the underlying social norm. We must however note that the observed effect sizes were small (cf., Tables 3 and 5).

In order to explore quantitatively our participants' behavior, we used a version of the Rescorla-Wagner algorithm to model performance in the Tipping Game. Modeling results show that differences in the attitude toward economic costs, captured by $w_{\text {econ }}$, could account for the behavioral differences much better than differences in outcome weights, rate of learning or action selection strategy.

Taken together, our findings confirm the potent role of social reinforcements on learning, which is predicted by classical social learning and facilitation theories (Zajonc, 1965; Bandura, 1977; see also Doris and Nichols, 2012). Consistent with previous findings (e.g., Hurlemann et al., 2010), social feedback in the form of facial expressions, if compared to non-social feedback in the form of conventional feedback marks, led people to be more generous. Furthermore, both behavioral and modeling results confirmed that social reward in the form of facial expressions bias decision-making, which is consistent with previous behavioral and modeling results focused on smiling facial expressions (Averbeck and Duchaine, 2009; Shore and Heerey, 2011). Specifically, our results extend the literature on the impact of subtle cues on social behavior (e.g., Bargh and Williams, 2006) indicating that emotional facial expression may bias behavior toward more generosity through lowering one's sensitivity to economic costs.

Angry facial expressions, in particular, might drive such a pattern of learning and decision-making by affecting the motivation to spend extra monetary units during a social interaction, thereby displaying more generous behavior. Angry facial expressions might signal social disapproval of a failure to comply with a norm. Such a failure might be due to a lack of knowledge of the social environment. Thus, learners of a social norm might feel anxious and uncomfortable in observing an angry reaction, which might in turn lead them to behave more generously in an attempt to avoid social disapproval in the future (Fehr and Gächter, 2002; Haley and Fessler, 2005; Bateson et al., 2006; Rigdon et al., 2009). Interestingly, the desire to avoid social disapproval is one of the main factors that apparently motivate people to tip in restaurants in the real world (Conlin et al., 2003; Azar, 2007).
Given the structure of the Tipping Game, and given that our Rescorla-Wagner model nicely fitted participants' choices, it is plausible that our task engaged a basic reinforcement learning mechanism, which is responsible for several social behaviors (Frith and Frith, 2012). According to reinforcement learning models (Sutton and Barto, 1998), estimated value of states or actions is updated through prediction error signals, whether about primary reward, money, or conventional or social choice feedback. These error signals have been found to accurately predict neural responses in the mesostriatal dopaminergic system and its targets (Montague et al., 1996; Niv, 2009; Colombo, 2014). Some findings suggest that both social and non-social reward are processed by the same striatal neural systems (Lin et al., 2012a), while other studies emphasize the specific role of mentalizing networks, associated with the temporoparietal junction, in processing social reward (e.g., Evans et al., 2011). In light of these results, one question for further research is whether a domain general, dopamine-based reinforcement learning mechanism can be sufficient for the acquisition of social norms (Ruff and Fehr, 2014). Examining results from the Tipping Game task using functional magnetic resonance imaging (fMRI) can be one way to make progress on that question.

Three limitations of our study should be singled out. First, some neurodevelopmental disorders, including autism, Asperger syndrome and schizophrenia, are known to affect the relative power of social vs. non-social feedback (cf., Montague et al., 2012). Furthermore, previous experiments suggest that males and females process some types of social reward differently (Spreckelmeyer et al., 2009). While we measured some scores relevant to assess autistic traits in Experiment 1, we did not collect them in Experiment 2, and we did not measure other personality traits potentially relevant to social decision-making and learning. Based on the scores that we did collect, and based on analyses of gender differences, the hypotheses that the behavioral effects we found could be explained solely by a gender difference or by some autistic trait in our participants could be ruled out. However, the issues remain to what extent gender differences in reward processing are associated with differences in social learning and decision-making, and how the social dysfunctions observed in people with certain neurodevelopmental disorders is linked to a specific deficit in the processing of social feedback.

Second, one reason why our participants did not generally perform well in the Tipping Game might be that its feedback structure made the learning task especially hard. Overall, the level of noise in the mapping between state-action pairs and reward outcomes was high across the blocks in our task, making the feedback provided not very reliable. Moreover, the reliability of the feedback was independent from the difference between amount tipped and underlying social norm, so that tips well above the social norm could still receive negative feedback. In order to improve learning performance, the feedback structure of the task may be modified in two ways. On the one hand, the level of noise in the mapping between state-action pairs and reward outcomes may be diminished across all blocks, similarly to what we did in the fourth block of Experiment 2. On the other hand, the reliability of the 
feedback provided may be made dependent on the distance between amount tipped and underlying social norm, so as to strengthen the reliability of feedback for tips well above or well below the social norm. Although our main behavioral results were robust to different levels of noise, it remains a question for further research how exactly social decisionmaking and learning are affected by the uncertainty of feedback information.

The third limitation concerns the distinction between social and emotional cues. The stimuli that we used in the social condition of our task did not help us to determine whether the behavioral effects we observed depended on social rather than on only the emotional dimension of facial expressions. Facial expressions convey both social and emotional information. Besides communicating information about other agents, facial expressions can often elicit emotional reactions in the observers (Furl etal., 2012). In order to identify the role of emotional cues alone, in contrast to facial expressions, on participants' learning and social decision-making, a third condition for our task may employ emotional, non-social reward. Further work will aim at clarifying these issues, and the generality of our findings.

\section{ACKNOWLEDGMENTS}

We are grateful to Luigi Acerbi, Bruno Averbeck, Jun Lai, Aaron Seitz, and Vincent Valton for useful discussions and feedback on previous drafts of this paper. Matteo Colombo was supported by the Deutsche Forschungsgemeinschaft (DFG) as part of the priority program "New Frameworks of Rationality" ([SPP 1516]). Aistis Stankevicius was supported in part by grants EP/F500385/1 and BB/F529254/1 for the University of Edinburgh School of Informatics Doctoral Training Centre in Neuroinformatics and Computational Neuroscience, from the UK Engineering and Physical Sciences Research Council (EPSRC), UK Biotechnology and Biological Sciences Research Council (BBSRC), and the UK Medical Research Council (MRC). The funders had no role in study design, data collection and analysis, decision to publish, or preparation of the manuscript.

\section{REFERENCES}

Adolphs, R. (2009). The social brain: neural basis of social knowledge. Annu. Rev. Psychol. 60, 693-716. doi: 10.1146/annurev.psych.60.110707. 163514

Allport, F. H. (1920). The influence of the group upon association and thought. J. Exp. Psychol. 3, 159-182. doi: 10.1037/h0067891

Averbeck, B. B., and Duchaine, B. (2009). Integration of social and utilitarian factors in decision making. Emotion 9, 599-608. doi: 10.1037/ a0016509

Azar, O. H. (2007). Why pay extra? Tipping and the importance of social norms and feelings in economic theory. J. Socio Econ. 36, 250-265. doi: 10.1016/j.socec.2005.11.046

Bandura, A. (1977). Social Learning Theory. Englewood Cliffs, NJ: Prentice-Hall.

Bargh, J. A., and Williams, E. L. (2006). The automaticity of social life. Curr. Dir. Psychol. Sci. 15, 1-4. doi: 10.1111/j.0963-7214.2006.00395.x

Baron-Cohen, S., and Wheelwright, S. (2004). The empathy quotient (EQ). An investigation of adults with Asperger syndrome or high functioning autism, and normal sex differences. J. Autism Dev. Disord. 34, 163-175. doi: 10.1023/B:JADD.0000022607.19833.00

Baron-Cohen, S., Wheelwright, S., and Jolliffe, T. (1997). Is there a "language of the eyes"? Evidence from normal adults and adults with autism or Asperger syndrome. Vis. Cogn. 4, 311-331. doi: 10.1080/713756761
Bateson, M., Nettle, D., and Roberts, G. (2006). Cues of being watched enhance cooperation in a real-world setting. Biol. Lett. 2, 412-414. doi: 10.1098/rsbl.2006.0509

Becker, G. (1976). The Economic Approach to Human Behavior. Chicago: University of Chicago Press.

Bicchieri, C. (2006). The Grammar of Society: The Nature and Dynamics of Social Norms. New York, NY: Cambridge University Press.

Blair, R. J. (2003). Facial expressions, their communicatory functions, and neurocognitive substrates. Philos. Trans. R. Soc. Lond. B Biol. Sci. 358, 561-572. doi: 10.1098/rstb.2002.1220

Brainard, D. H. (1997). The Psychophysics Toolbox. Spat. Vis. 10, 433-436. doi: $10.1163 / 156856897 X 00357$

Carver, C. S., and White, T. L. (1994). Behavioral inhibition, behavioral activation, and affective responses to impending reward and punishment: the BIS/BAS scales. J. Pers. Soc. Psychol. 67, 319-333. doi: 10.1037/0022-3514. 67.2.319

Colombo, M. (2014). Deep and beautiful. the reward prediction error hypothesis of dopamine. Stud. Hist. Philos. Biol. Biomed. Sci. 45, 57-67. doi: 10.1016/j.shpsc.2013.10.006

Conlin, M., Lynn, M., and O’Donoghue, T. (2003). The norm of restaurant tipping. J. Econ. Behav. Organ. 5, 297-321. doi: 10.1016/S0167-2681(03)00030-1

Doris, J. M., and Nichols, S. (2012). "Broadminded: sociality and the cognitive science of morality," in The Oxford Handbook of Philosophy and Cognitive Science, eds E. Margolis, R. Samuels, and S. Stich (Oxford: Oxford University Press), 425-453.

Ernest-Jones, M., Nettle, D., and Bateson, M. (2011). Effects of eye images on everyday cooperative behavior: a field experiment. Evol. Hum. Behav. 32, 172-178. doi: 10.1016/j.evolhumbehav.2010.10.006

Evans, S., Fleming, S. M., Dolan, R. J., and Averbeck, B. B. (2011). Effects of emotional preferences on value-based decision-making are mediated by mentalizing and not reward networks. J. Cogn. Neurosci. 23, 2197-2210. doi: 10.1162/jocn.2010.21584

Fehr, E., and Gächter, S. (2002). Altruistic punishment in humans. Nature 415, 137-140. doi: 10.1038/415137a

Frith, C. (2009). Role of facial expressions in social interactions. Philos. Trans. $R$. Soc. Lond. B 364, 3453-3458. doi: 10.1098/rstb.2009.0142

Frith, C., and Frith, U. (2012). Mechanisms of social cognition. Annu. Rev. Psychol. 63, 287-313. doi: 10.1146/annurev-psych-120710-100449

Furl, N., Gallagher, S., and Averbeck, B. B. (2012). A selective emotional decision-making bias elicited by facial expressions. PLOS ONE 7:e33461. doi: 10.1371/journal.pone.0033461

Haley, K. J., and Fessler, D. (2005). Nobody's watching? Subtle cues affect generosity in an anonymous dictator game. Evol. Hum. Behav. 26, 245-256. doi: 10.1016/j.evolhumbehav.2005.01.002

Hurlemann, R., Patin, A., Onur, O. A., Cohen, M. X., Baumgartner, T., Metzler, S., et al. (2010). Oxytocin enhances amygdala dependent, socially reinforced learning and emotional empathy in humans. J. Neurosci. 30, 4999-5007. doi: 10.1523/JNEUROSCI.5538-09.2010

Keltner, D., and Haidt, J. (1999). The social functions of emotions at multiple levels of analysis. Cogn. Emot. 13, 505-522. doi: 10.1080/026999399379168

Kleiner, M., Brainard, D., and Pelli, D. (2007). What's new in 8Psychtoolbox-3? Perception 36, ECVP Abstract Supplement.

Lin, A., Adolphs, R., and Rangel, A. (2012a). Social and monetary reward learning engage overlapping neural substrates. Soc. Cogn. Affect. Neurosci. 7, 274-281. doi: 10.1093/scan/nsr006

Lin, A., Rangel, A., and Adolphs, R. (2012b). Impaired learning of social compared to monetary rewards in autism. Front. Neurosci. 6:143. doi: 10.3389/fnins.2012.00143

Lyons, M. J., Akamatsu, S., Kamachi, M., and Gyoba, J. (1998). "Coding facial expressions with Gabor wavelets," in Proceedings of the Third IEEE International Conference on Automatic Face and Gesture Recognition, April 14-16 1998, (Nara: IEEE Computer Society), 200-205. doi: 10.1109/AFGR.1998. 670949

Montague, P., Dayan, P., and Sejnowski, T. J. (1996). A framework for mesencephalic dopamine systems based on predictive hebbian learning. J. Neurosci. 16, 19361947.

Montague, P. R., Dolan, R. J., Friston, K. J., and Dayan, P. (2012). Computational psychiatry. Trends Cogn. Sci. 16, 72-80. doi: 10.1016/j.tics.2011.11.018 
Niv, Y. (2009). Reinforcement learning in the brain. J. Math. Psychol. 53, 139-154. doi: 10.1016/j.jmp.2008.12.005

Oaksford, M., and Chater, N. (2007). Bayesian Rationality: The Probabilistic Approach to Human Reasoning. Oxford: Oxford University Press. doi: 10.1093/acprof:oso/9780198524496.001.0001

Pelli, D. G. (1997). The VideoToolbox software for visual psychophysics: transforming numbers into movies. Spat. Vis. 10, 437-442. doi: 10.1163/156856897 X00366

Reeb-Sutherland, B. C., Levitt, P., and Fox, N. A. (2012). The predictive nature of individual differences in early associative learning and emerging social behavior. PLOS ONE 7:e30511. doi: 10.1371/journal.pone. 0030511

Rescorla, R. A., and Wagner, A. R. (1972). "A theory of Pavlovian conditioning: Variations in the effectiveness of reinforcement and nonreinforcement," in Classical Conditioning II: Current Research and Theory, eds A. H. Black and W. F. Prokasy (New York, NY: Appleton Century Crofts), 64-99.

Rigdon, M., Ishii, K., Watabe, M., and Kitayama, S. (2009). Minimal social cues in the dictator game. J. Econ. Psychol. 30, 358-367. doi: 10.1016/j.joep.2009. 02.002

Ruff, C. C., and Fehr, E. (2014). The neurobiology of rewards and values in social decision making. Nat. Rev. Neurosci. 15, 549-562. doi: 10.1038/nrn3776

Rushton, J. P., Chrisjohn, R. D., and Fekken, G. C. (1981). The altruistic personality and the self-report altruism scale. Pers. Individ. Dif. 2, 293-302. doi: 10.1016/0191-8869(81)90084-2

Seidel, E. M., Habel, U., Kirschner, M., Gur, R. C., and Derntl, B. (2010). The impact of facial emotional expressions on behavioral tendencies in women and men. J. Exp. Psychol. Hum. Percept. Perform. 36, 500-507. doi: 10.1037/ a0018169

Shore, D. M., and Heerey, E. A. (2011). The value of genuine and polite smiles. Emotion 11, 169-174. doi: 10.1037/a0022601
Spreckelmeyer, K. N., Krach, S., Kohls, G., Rademacher, L., Irmak, A., Konrad, K., et al. (2009). Anticipation of monetary and social reward differently activates mesolimbic brain structures in men and women. Soc. Cogn. Affect. Neurosci. 4, 158-165. doi: 10.1093/scan/nsn051

Sutton, R. S., and Barto, A. G. (1998). Reinforcement Learning: An Introduction. Cambridge, MA: MIT Press.

Torrubia, R., Àvila, C., Moltò, J., and Caseras, X. (2001). The Sensitivity to Punishment and Sensitivity to Reward Questionnaire (SPSRQ) as a measure of Gray's anxiety and impulsivity dimensions. Pers. Individ. Dif. 31, 837-862. doi: 10.1016/S0191-8869(00)00183-5

Zajonc, R. B. (1965). Social facilitation. Science 149, 269-274. doi: 10.1126/science.149.3681.269

Conflict of Interest Statement: The authors declare that the research was conducted in the absence of any commercial or financial relationships that could be construed as a potential conflict of interest.

Received: 25 April 2014; paper pending published: 30 July 2014; accepted: 23 September 2014; published online: 09 October 2014

Citation: Colombo M, Stankevicius A and Seriès P (2014) Benefits of social vs. nonsocial feedback on learning and generosity. Results from the Tipping Game. Front. Psychol. 5:1154. doi: 10.3389/fpsyg.2014.01154

This article was submitted to Cultural Psychology, a section of the journal Frontiers in Psychology.

Copyright (C) 2014 Colombo, Stankevicius and Seriès. This is an open-access article distributed under the terms of the Creative Commons Attribution License (CC BY). The use, distribution or reproduction in other forums is permitted, provided the original author(s) or licensor are credited and that the original publication in this journal is cited, in accordance with accepted academic practice. No use, distribution or reproduction is permitted which does not comply with these terms. 